\title{
COMPARACIÓN DEL ESFUERZO CARDIOVASCULAR EN LA UTILIZACIÓN DE LA MÁQUINA HEALTH WALKER Y EL EJERCICIO DE CAMINAR EN ADULTOS SANOS
}

\author{
Pietro Scaglioni Solano y Luis Fernando Aragón Vargas \\ Laboratorio de Rendimiento Físico y Salud (LARENFISA) \\ Escuela de Educación Física y Deportes \\ Universidad de Costa Rica, San José, Costa Rica \\ E-mail:pscaglio@cariari.ucr.ac.cr
}

\begin{abstract}
Resumen
Scaglioni Solano, P. y Aragón Vargas, L. F. (2001). Comparación del esfuerzo cardiovascular en la utilización de la máquina Health Walker y el ejercicio de caminar en adultos sanos. Revista de Ciencias del Ejercicio y la Salud, 1(1), 11-18. Este estudio comparó el uso de la máquina Health Walker, que simula caminar con un movimiento pendular y que tiene dos opciones de posicionamiento de brazos para tener dos modos de ejercicio distintos, con el ejercicio de caminar, mediante la medición de la frecuencia cardíaca, a valores similares de cadencia (3 niveles: 100,120,138 pasos/min). También se estudiaron valores de autoregulación en el ejercicio (cadencia natural y medición de frecuencia cardíaca para esa cadencia). 15 mujeres (59.8+-4.8años) realizaron las pruebas, administradas aleatoriamente en sus tres modalidades (caminar, ejercicio en máquina con brazos en posición fija, máquina con brazos en posicionamiento móvil), con aumento gradual de la cadencia, y durante cinco minutos en cada uno de los tres valores de ésta. Para la cadencia natural, se indicó a la persona que realizara un calentamiento de cinco minutos antes de cada prueba, donde se hicieron observaciones sin previo aviso. Los resultados obtenidos indican que la interacción entre el modo de ejercicio y la cadencia produce diferencias significativas $(p<0.001)$ en el valor de la frecuencia cardíaca, lo que indica que cada modo de ejercicio responde de manera distinta a los cambios en el valor de la cadencia. Así, se puede concluir que el uso de la máquina Health Walker con el modo de doble acción (brazos y piernas) es similar al ejercicio de caminar en cuanto a esfuerzo cardiovascular. Para la parte de autoregulación, se encontró que los sujetos "regularon" su ejercicio con base en un esfuerzo similar en cuanto a frecuencia cardiaca (cerca de 100 lat. $\cdot$ min $^{-1}$ (60\% de la FCmáx), mientras que los valores de cadencia natural sí mostraron diferencias significativas $(p<0.01)$ entre caminar y el modo en la máquina con los brazos fijos. PALABRAS CLAVES: caminar, frecuencia cardiaca, ejercicio de brazos y piernas, cadencia natural.
\end{abstract}

\section{INTRODUCCION}

El ejercicio de caminar se ha popularizando entre la población adulta como una forma de ejercicio alternativo para cumplir sus necesidades de actividad física que se puede desarrollar con una intensidad moderada y con el mínimo estrés osteomuscular. Muchos médicos y profesionales del ejercicio recomiendan caminar regularmente como una forma de ejercicio. Aparentemente muchos adultos actúan según este consejo pero poco se sabe acerca de cómo lo están poniendo en práctica (Spelman, Pate, Macera y Ward;1993).

El proceso de caminar. El término caminar no es específico. Su connotación es la de un patrón cíclico de movimientos del cuerpo que se repite una y otra vez, paso a paso. Consecuentemente, las descripciones que se hacen del acto de caminar tratan con lo que pasa en el curso de un ciclo, asumiendo que los ciclos sucesivos serán prácticamente iguales (Inman, Ralston y Todd; 1981). En realidad, caminar es un fenómeno complejo, y una adecuada descripción de lo que ocurre durante 
un ciclo requiere la consideración de factores tales como reacciones sobre el piso, requerimientos energéticos totales, transferencias energéticas dentro del cuerpo y la acción muscular (Inman et al.,1981).

Medidas temporales de zancada o paso. La unidad fundamental de la fase rítmica de cualquier forma de locomoción que hace uso de las piernas es la zancada o ciclo básico de caminar, que se define como la secuencia de movimientos que ocurre entre dos repeticiones consecutivas de la configuración de un cuerpo (Inman et al.,1981).

Un ciclo tiene dos dimensiones: el período de tiempo desde el inicio al final, y la distancia que el cuerpo se mueve durante un ciclo (largo de zancada). La duración temporal se expresa generalmente por su recíproco, la frecuencia de zancada (cadencia). Para cambiar la velocidad al caminar se puede variar el largo o la cadencia, y una persona normal varía los dos. Típicamente, la gráfica de largo de zancada contra frecuencia tiende a crecer en el rango de velocidades normales de 70 a 130 pasos/min., nivelándose a velocidades altas en una longitud constante (Inman et al.,1981), como se aprecia en la figura 1.

Figura 1

Variación de la zancada

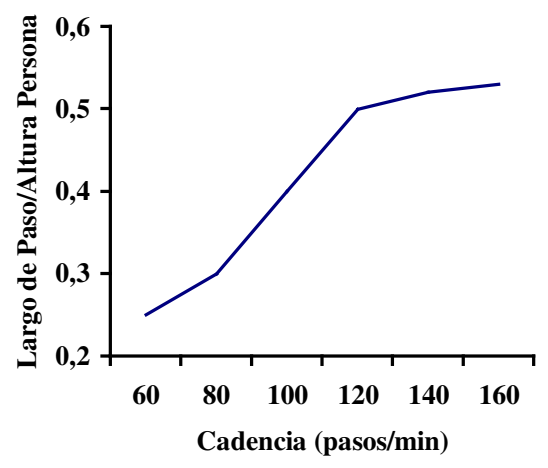

La persona normal regresará consistentemente al mismo patrón cuando se le prueba en repetidas ocasiones, mientras no haya cambios en el calzado o en la superficie sobre la que se camina, lo que podría traer cambios en cadencia o zancada (Inman et al., 1981).

Velocidades al caminar: Cada persona es capaz de caminar a distintas velocidades, y adoptará en cualquier momento una velocidad, de lenta a rápida, que llene sus necesidades en ese momento.

Cadencia natural: Se define como el número de pasos/min. que un sujeto hace caminando de la forma más natural posible. Cuando se camina más despacio o más rápido se debe cambiar nuestra frecuencia natural y conscientemente forzar la cadencia a una razón más rápida o más lenta (Winter,1991).

Cadencias en adultos: Los estudios de transeúntes urbanos indican que rara vez un hombre adulto camina a una frecuencia fuera del rango de 75 a 140 pasos/min. El rango equivalente para mujeres es de 80-150 pasos/min. La parte central del rango, de 100 a 120 pasos/min., corresponde un valor de paso de velocidad media para un hombre adulto. Para mujeres el valor comparable es de 5 pasos/min. por encima (Inman et al.,1981).

Mientras caminar y trotar son las formas más ampliamente utilizadas de ejercicio aeróbico, algunas personas son incapaces de correr debido a problemas ósteomusculares. Además muchos entusiastas del ejercicio buscan alternativas atractivas para complementar los programas de caminar y trotar, que activan principalmente la parte inferior del cuerpo.

Debido a los beneficios que trae la práctica regular de ejercicio aeróbico (American College of Sports Medicine, 1990 y 1991) para desarrollar y mantener la buena condición cardiorespiratoria y muscular y la salud en general, recientemente se ha popularizado la utilización de máquinas especialmente diseñadas para la actividad física y que desarrollan una forma de ejercicio para todo el cuerpo, como por ejemplo las simuladoras de esquí de fondo (Goss, Robertson y Spina; 1989). También existen otros tipos de máquinas que pretenden cumplir estas finalidades, pero no se tiene referencia acerca de sus efectos metabólicos y 
biomecánicos, y es parte de la inquietud de este estudio.

En este caso se estudió la máquina Health Walker, que es el nombre comercial de un aparato que ofrece varias modalidades de uso:

\section{Figura 2}

Máquina Health Walker

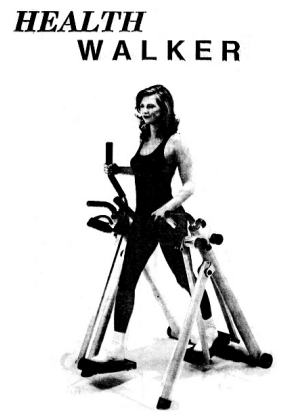

La primera modalidad es simular el ejercicio de caminar, sin impacto en rodillas y pies (posición fija de agarraderas para manos) y la segunda modalidad es un ejercicio combinado de piernas y brazos al utilizar el modo de doble acción con agarraderas móviles para las manos, que van unidas a las estructuras móviles para las piernas.

También existen otros aparatos similares de otros fabricantes, con diferentes nombres comerciales como por ejemplo: "Ski Flyer", de la fábrica NordicTrack, o el "Space Walker" de Interwood.

Vale la pena mencionar aquí que esta máquina pretende simular el ejercicio de caminar con un movimiento pendular periódico de amplitud variable y que en dicho movimiento no hay flexión ni extensión de rodillas, sino solamente flexión y extensión de caderas. Tampoco hay flexión plantar ni dorsiflexión, lo que en realidad hace que este ejercicio tenga algunas diferencias con respecto a caminar, desde el punto de vista de la biomecánica, la técnica de ejecución y los grupos musculares utilizados.

Este aparato también tiene un control de ajuste de tensión que aumenta o disminuye la resistencia al ejercitarse en la máquina, lo que hace posible una serie de combinaciones de ejercicios e intensidades.

Este tipo de equipo ha sido diseñado más bien para uso recreativo y deportivo y no como ergómetro de laboratorio para investigación, por lo que la estimación de consumos de energía en unidades de trabajo fisiológico no es posible. De todas maneras la información que se pueda obtener de acuerdo a un cierto volumen e intensidad de trabajo es valiosa para evaluar este tipo de ejercicio (Goss et al., 1989).

Este estudio se centró más bien en la parte de requerimientos energéticos reflejados en cambios fisiológicos (frecuencia cardíaca), donde se comparó el esfuerzo cardiovascular que representa la utilización de la máquina Health Walker con el ejercicio de caminar, partiendo de la hipótesis que el esfuerzo es similar para un valor dado de cadencia, en las tres modalidades de ejercicio (caminar, máquina con brazos fijos, máquina con brazos en movimiento). Como parámetro de comparación se utilizó la frecuencia cardíaca.

Como segunda hipótesis se planteó que la utilización de la máquina Health Walker bajo condiciones de autoregulación es diferente en cuanto a cadencia, pero similar en esfuerzo cardíaco, con respecto a caminar, y que la intensidad de este ejercicio es suficiente para cumplir con la intensidad recomendada por el Colegio Americano de Medicina Deportiva, (American College of Sports Medicine, 1990) para desarrollar y mantener la buena condición cardiorespiratoria y muscular (intensidad aproximada desde un $60 \%$ al $90 \%$ de la FCmáx).

La estimación de intensidades autoseleccionadas de ejercicio son esenciales para la investigación epidemiológica por dos razones. Primero, la intensidad autoseleccionada se debe determinar para calcular gastos energéticos. Segundo, la información acerca de la intensidad autoseleccionada para el ejercicio, tanto al caminar como en la máquina, se pueden usar en conjunto con datos de salud para ayudar a aclarar la relación entre la intensidad del ejercicio y la salud. Adicionalmente, el 
conocimiento de los niveles autoseleccionados de esfuerzo (junto con datos de frecuencia y duración del ejercicio escogido) pueden proveer una visión que ayude al público a interpretar las guías de prescripción de ejercicio, y permitir a los médicos y educadores físicos brindar un mensaje más efectivo.

Por otro lado, la comparación de modos de ejercicio (caminar y ejercicio en máquina) brinda una información valiosa al público que se interesa por el uso de este tipo de aparatos, diseñados para simular una actividad específica y de los cuales no se tiene información más detallada de su desempeño.

\section{METODOLOGIA}

\section{Sujetos}

De un grupo de actividad física para la tercera edad de la Universidad de Costa Rica, se escogieron 15 mujeres que sirvieron como sujetos para este estudio. En una entrevista previa se obtuvo información personal sobre posibles limitaciones y/o riesgos para efectuar este tipo de pruebas submáximas. Se encontraron casos de hipertensión controlada, y recuperación completa de enfermedad cardiovascular. Cada sujeto llenó una fórmula de consentimiento donde se explicó el propósito y métodos del experimento.

Tabla 1

Características descriptivas del grupo de estudio $(n=15)$

\begin{tabular}{lc}
\hline \multicolumn{1}{c}{ Variable } & M $\pm(\mathbf{D S})$ \\
\hline Edad (años) & $59.8(4.8)$ \\
Peso $(\mathrm{Kg})$ & $67.9(11.5)$ \\
Altura $(\mathrm{cm})$ & $156.6(4.9)$ \\
$\mathrm{IMC}\left(\mathrm{kg} / \mathrm{m}^{2}\right)^{*}$ & $27.4(4.1)$ \\
$\mathrm{FC}_{\text {máx }} * *$ & $171.2(4.3)$ \\
$60 \% \mathrm{FC}_{\text {máx }}$ & $102.7(2.5)$ \\
\hline
\end{tabular}

$>$ Todos los valores son promedios (M) y desviaciones estándar (DS)

* Indice de masa corporal= Peso/estatura2

** Frecuencia Cardíaca máxima según la

fórmula: $\mathrm{FC}_{\text {máx }}=219-0.8 *$ edad

\section{Instrumentos de medición}

Para realizar las pruebas se requirió de los siguientes equipos:

- Máquina Health Walker

- Monitor Polar, modelo Pacer, para monitorear la frecuencia cardíaca.

- Cronómetro

- Metrónomo Yamaha MP-70, el cual se utilizó para grabar una cinta con los tres valores de cadencia utilizados

- Grabadora

- Cancha de tenis (o sección de terreno plano) para las pruebas de caminar

\section{Procedimientos de medición}

De una prueba piloto se definieron los valores para la cadencia y el tiempo para cada prueba, de acuerdo a las intensidades de ejercicio (que no representaran un esfuerzo mayor que el $80 \%$ de la frecuencia cardíaca máxima) y a la posibilidad de coordinación de los movimientos, tanto en la máquina como a pie. Se siguieron las recomendaciones descritas en la literatura acerca de valores de cadencias al caminar para mujeres, y se definieron tres valores de cadencia: 100120 y 138 pasos $\cdot \min ^{-1}$ (Inman et al., 1981; Winter, 1991)

De esta prueba piloto se fijó el valor de resistencia de la máquina al mínimo, debido a la incapacidad de regular o ajustar la misma con cierto grado de precisión.

Antes de las pruebas los sujetos practicaron en la máquina unas 3-4 veces durante 5-10 min. para familiarizarse con el uso en las dos opciones de posicionamiento de brazos.

Pruebas experimentales: Las pruebas consistieron en medir la frecuencia cardíaca del sujeto en las 3 modalidades de ejercicio, asignando aleatoriamente el orden de aplicación de los tratamientos (caminar, ejercicio en la máquina con brazos fijos, ejercicio en la máquina con brazos en movimiento). 


\section{Análisis estadístico}

ANOVA de dos vías, $3 \times 3$ (variables independientes: cadencia y modo, con 3 niveles para cada uno) para las pruebas con valores definidos de cadencia. La variable dependiente es la frecuencia cardíaca.

ANOVA de una vía con 3 niveles (variable independiente: Modo de ejercicio, 3 modos), para la cadencia natural (variable dependiente: Cadencia).

ANOVA de una vía con 3 niveles (variable independiente: los 3 modos de ejercicio) para los valores de frecuencia cardíaca en la cadencia natural (variable dependiente: Frecuencia cardíaca).

Análisis post-hoc de Newman-Keuls para la cadencia natural.

El valor de significancia se estableció a priori en el valor de 0.05 para todos los análisis.

\section{RESULTADOS}

Datos descriptivos: Se muestra en la tabla 2 el resumen de características físicas de los sujetos.

Para la parte de comparación de los modos de ejercicio bajo los tres valores de cadencia preestablecidos $(100,120,138$ pasos $\left.\cdot \min ^{-1}\right)$, la interacción entre el modo de ejercicio y la cadencia (cambio en ambas variables) fue significativa $(p<0.001)$ en el valor de la frecuencia cardíaca, lo que se puede interpretar como que al menos un modo de ejercicio responde de manera diferente a los cambios en el valor de la cadencia (Figura 3). Esto se refleja en las inclinaciones distintas en las pendientes del gráfico, tanto para cada modo de ejercicio en las distintas cadencias como para los modos en cada valor de cadencia.

Tabla 2

Frecuencia cardíaca para modos de ejercicio en cadencias prefijadas

\begin{tabular}{llccc}
\hline \multicolumn{1}{c}{ Modo } & & \multicolumn{3}{c}{ Cadencia } \\
\cline { 3 - 5 } Caminar & & $\mathbf{1 0 0}$ & $\mathbf{1 2 0}$ & $\mathbf{1 3 8}$ \\
& Promedio & 93.6 & 99.1 & 103.9 \\
Máquina brazos fijos & Desviación estándar & 10.9 & 12.1 & 13.9 \\
\cline { 2 - 5 } Máquina brazos movimiento & Promedio & 92.2 & 92.4 & 95.8 \\
& Desviación estándar & 10.1 & 11.3 & 9.9 \\
\cline { 2 - 5 } & Promedio & 96.7 & 101.9 & 104.1 \\
& Desviación estándar & 13.3 & 17.4 & 16.1 \\
\hline
\end{tabular}

Comparando el modo de caminar con el modo de la máquina con brazos en posición fija (MBF) para valores bajos de cadencia no hay diferencias significativas en la frecuencia cardíaca, pero al aumentar la cadencia, la diferencia en esfuerzo empieza a ser significativa.

Comparando ahora el modo de caminar con el modo de la máquina con brazos en movimiento (MBM), la diferencia en frecuencia cardíaca a valores bajos de cadencia es mayor que cuando aumenta la cadencia, tanto que al final prácticamente no hay diferencia.

Para el menor valor de la cadencia (100 pasos $\bullet \min ^{-1}$ ) (Fig. 3), las diferencias entre modos no son significativas. Para los otros valores de cadencia (120 y 138 pasos • $\min ^{-1}$, respectivamente) (Fig. 3) las diferencias son significativas entre los modos de Caminar y MBM con respecto al modo MBF. 
Figura 3

Comparación frecuencia cardíaca en modos a cadencias prefijadas

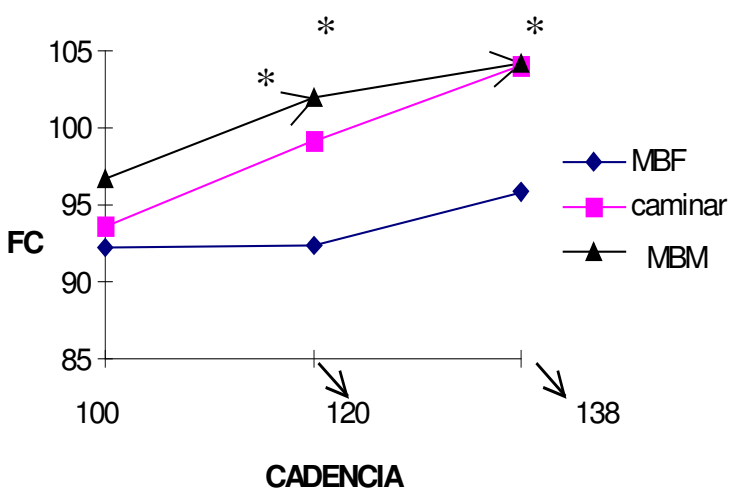

$\mathrm{MBF}$ : modo máquina brazos fijos

MBM modo máquina brazos en movimiento

$* p<0.001$, diferencia en FC entre Caminar y MBM con respecto a MBF

Para la segunda parte de esta investigación, se presentan los resultados en las figuras 4 y 5: La figura 4 indica que existe diferencia significativa $(p<0.01)$ para el valor de cadencia natural entre caminar y el modo de la máquina con brazos en posición fija. La figura 5 indica que la frecuencia cardíaca durante la cadencia natural no presentó diferencias significativas entre los tres modos de ejercicio.

Figura 4 Cadencia en autorregulación

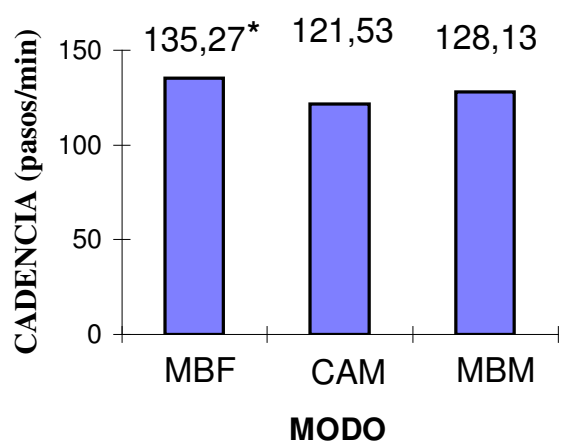

$* p<0.01$, diferencia entre CAM y MBF

\section{DISCUSION}

La comparación de modos a valores prefijados de cadencia da información sobre el rendimiento de la máquina Health Walker con respecto al esfuerzo cardiovascular durante el ejercicio.

Figura 5

Frecuencia cardíaca en autorregulación

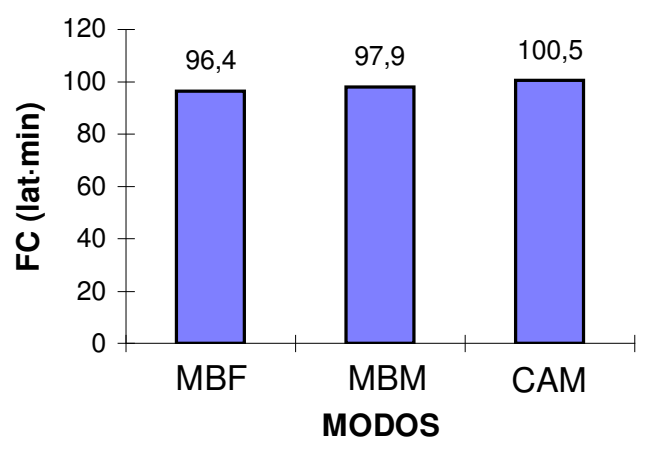


Los resultados indican que bajo las condiciones de prueba, el uso de la máquina con los brazos en la posición móvil se puede comparar con el ejercicio de caminar en cuanto a esfuerzo, mientras que entre el uso de la máquina con los brazos en la posición fija y el ejercicio de caminar se notan diferencias significativas a partir del primer valor de cadencia $\left(100\right.$ pasos $\bullet \min ^{-1}$ ). Esto indica que el ejercicio en la máquina no simula el ejercicio de caminar bajo esta modalidad de uso.

Aquí es importante notar que el valor de la resistencia prefijado fue el mínimo, así que es posible que al aplicar una cierta carga de resistencia los valores de esfuerzo se puedan igualar entre estos modos de ejercicio.

Comparando los modos de ejercicio de la máquina se nota que existen diferencias significativas entre sí, y que el mayor esfuerzo cardiovascular se debe a la utilización de más grupos musculares en la posición de los brazos en las agarraderas móviles, lo que implica un modo de ejercicio más completo y de mayor gasto energético.

Para la segunda parte de la investigación cabe destacar que los valores obtenidos para la cadencia natural al caminar están dentro del rango de los valores observados en la literatura, (105 a 125 pasos - $\min ^{-1}$ ) para mujeres adultas (Inman et al. 1981; Winter.1991).

En esta investigación se encontró que la persona autoregula su esfuerzo a una intensidad similar en los tres modos de ejercicio, que se refleja en una frecuencia cardíaca similar.

Para regular este esfuerzo, el sujeto provoca variaciones en la cadencia dependiendo de la modalidad de ejercicio. Entre caminar y el modo en la máquina con brazos en posición fija la diferencia fue significativa en cuanto a este valor de cadencia natural, siendo mayor al ejercitarse en la máquina.

La diferencia en los valores de cadencia natural podría estar relacionada con la diferencia en cuanto a patrón biomecánico y activación muscular durante la ejecución de cada uno de los tres modos de ejercicio.

Los valores de frecuencia cardíaca durante el ejercicio autoregulado (Fig.5), están muy cercanos al valor de intensidad de un $60 \%$ de la frecuencia cardíaca máxima (Tabla1: 102.7 lat $\bullet \mathrm{min}^{-1}$ ), lo que hace que el ejercicio autoregulado en estas modalidades se acerque al límite del rango recomendado por el ACSM para desarrollar y mantener la buena condición cardiorespiratoria y muscular en adultos sanos (60-90\% de FCmáx).

Aquí es importante mencionar que el valor de frecuencia cardíaca máxima se obtiene a través de una fórmula predictiva, por lo tanto es un valor que varía de sujeto a sujeto, y también depende de la fórmula de predicción utilizada, ya que si se utiliza la fórmula de 220- edad, se obtendría un valor de 96 lat $\cdot \min ^{-1}$, y de esta manera se cumpliría que el valor de la frecuencia cardíaca durante la autoregulación está dentro del rango recomendado por el ACSM, que se mencionó anteriormente.

El ACSM reconoce los beneficios potenciales para la salud derivados de la práctica regular de ejercicio desarrollado con mayor frecuencia y durante más tiempo, pero a intensidades más bajas que las mencionadas en el párrafo anterior. (ACSM, 1991).

Se puede entonces concluir que los tres modos de ejercicio estudiados tienen una validez desde el punto de vista de ejercicio para salud, partiendo del hecho que la actividad desarrollada es suficiente para activar el sistema cardiovascular y muscular y obtener beneficios en cuanto a salud. Esto respaldado en el artículo del NIH Consensus Conference, acerca de la actividad física y la salud cardiovascular (NIH,1996) donde se menciona la importancia de la práctica regular de actividades físicas con intensidades moderadas para obtener beneficios de salud. Se habla de una actividad con una duración de unos 30 minutos acumulados durante el día, y cómo mucha gente no aprecia el valor de caminar 
como un "ejercicio" y no reconocen los beneficios substanciales de sesiones de corta duración (por lo menos 10 min.). También se respalda esta posición en el estudio de Blair, Kohl, Paffenbarger, Clark, Cooper y Gibbons (1989) acerca de la actividad física y las causas de mortalidad se mencionan algunos de estos beneficios, y para disminuir la tasa de mortalidad en mujeres, se recomienda una caminata briosa de 30 a $60 \mathrm{~min}$. cada día para lograr tener un nivel de actividad física que supere los bajos niveles que prevalecen en la población en general, y que hacen que el sedentarismo sea un problema de salud pública. (Blair, 1989).

Por otro lado, los modos de ejercicio se diferencian en cuanto a la respuesta cardiovascular a cadencias prefijadas de 120 y 138 entre los modos de caminar y MBM con respecto a MBF, lo cual se puede explicar por la diferencia en cuanto a la técnica de ejecución en cada modo de ejercicio y los grupos musculares utilizados en cada uno de ellos. Así, se observa que la respuesta cardiovascular para ejercicio autoregulado es similar en los tres modos de ejercicio a costa de cambios en la cadencia natural.

\section{REFERENCIAS}

American College of Sports Medicine. (1991). Guidelines for Graded Exercise Testing and Exercise Prescription. ( $4^{\text {th }}$.ed.). Pittsburgh: Williams \& Wilkins.

American College of Sports Medicine. (1990). Position Stand: The Recommended Quantity and Quality of Exercise for Developing and Maintaining Cardiorespiratory and Muscular Fitness in Healthy Adults. In Med.Sci.Sports Ex. 22, 265-274.

Aragón, L.F., Fernández, A.(1995). Fisiología del ejercicio. San José: Editorial Universidad de Costa Rica.

Blair, S.N., Kohl, H.W., Paffenbarger, R.S., Clark, D.G., Cooper, K.H. \& Gibbons, L.W. (1989). Physical fitness and all-cause mortality: a prospective study of healthy men and women. JAMA 262: 23952401.

Goss, F.L., Robertson, R.J., y R.J. Spina. (1989). Aerobic Metabolic Requirements of Simulated Cross Country Skiing. Ergonomics, 32 (12), 15731579.
Hardy, R. (1988).Self Regulation training in Sports and Work. Ergonomics, 31 (11), 1573-1583.

Inman, V.T., Ralston, H,J., y F. Todd. (1981). Human Walking. Baltimore: Williams \& Wilkins.

McArdle, W.D., Katch, F.L., and Katch, V.L.(1993). Exercise Physiology: Energy, Nutrition and Human Performance $\left(3^{\text {rd }} \cdot\right.$ de.). Pittsburgh: Human Kinetics.

N.I.H. (1996). Consensus Development Panel on Physical Activity and Cardiovascular Health Physical Activity and Cardiovascular Health. JAMA 276: 241-246.

Spelman, C.C., Pate, R.R., Macera, C.A., y D.S. Ward. (1993). Self Selected Exercise Intensity of Habitual Walkers. Med.Sci.Sports Exe., 25 (10), 11741179.

Vaugham, C.L., Davis, B.L., O'Connor, J.C. (1992). Dynamics of Human Gait. Champaign: Human Kintics publishers.

Winter, D.A. (1991). The Biomechanics and Motor Control of Human Gait: Normal, Elderly and Pathological. Ontario: University of Waterloo Press. 